\title{
Advanced Panspermia of Origin of Life and Evolution
}

\author{
Venkata Subba Rao Yeragudipati \\ University Science and Instrumentation Centre, Sri Venkateswara University, Tirupati, India
}

Email address:

yvsubbarao2004@yahoo.com

\section{To cite this article:}

Venkata Subba Rao Yeragudipati. Advanced Panspermia of Origin of Life and Evolution. American Journal of Astronomy and Astrophysics. Vol. 7, No. 4, 2019, pp. 54-66. doi: 10.11648/j.ajaa.20190704.12

Received: November 2, 2019; Accepted: November 21, 2019; Published: November 26, 2019

\begin{abstract}
In this study, a theory on Advanced Panspermia of Origin of Life and Evolution is proposed by establishing (i) striking planetary similarities observed for parents and their children of their respective birth times similar to matching bands in DNA Fingerprinting images of them establishing heredity; (ii) the number of DNA entries required to differentiate one person from the other being identical to the number of planetary configurations that would result (iii) the Lunar Magnetism Orchestrated Menstrual Cycle in Symphony of Reproduction of Life, and (iv) Cambrian Explosion witnessed origin of complex vertebrates simultaneously and all of a sudden. All the above comprise the strongest scientific evidence in the "Breakthrough of "Advanced Panspermia of Origin of Life and Evolution". Perhaps, the totipotent cell, formed in space under abiotic conditions during the early days of the solar system, picks up different magnetic signals from out of the solar symphony and at different stages of its development and the DNA is thus programmed. These pre-programmed magnetic memories may be evoked later in evolution when similar magnetic signals are repeated. Life came into being from space brought by star dust on the Earth with each major change of climate for evolution at intervals of simple multiples of Geological Time Scale 'Periods'. The study also discusses of how the Sun, Moon, and planets telegraph their effects to us, via interplanetary space striking Earth's magnetosphere, which are perceived by the neural network of the living beings, which are genetically predisposed to hear specific planetary signals.
\end{abstract}

Keywords: Panspermia, Totipotent Cell, DNA, Meteorite, Solar System, Interplanetary Magnetic Field, Earth's Magnetosphere, Geological Time Scale

\section{Introduction}

More than 97 percent of the mass of a human body is made up of carbon, hydrogen, nitrogen, oxygen, phosphorus and sulphur "CHNOPS" the six most common elements of life on Earth. This establishes an undeniable fact that most of the essential elements of life are made in stars. Synthesis of life building blocks including amino acids are formed under abiotic conditions. Star dust propel organisms over enormous distances between planets; Climate and environment have a great role in the evolution and extinction of species besides the breaking up and formation of supercontinents which appear to be cyclical; the successive pairs of planetary structure of Solar System gives rise to multiple DNA sequences of multiple species of myriad frequencies wherein the successive pairs of planets plausibly represent pairs of codon - amino acids circles; Electromagnetic force of synchronisation of successive pairs of planets of Solar System plausibly relate to three important aspects, namely, (i)
Major changes in climate and environment, (ii) Origin of new totipotent cells (iii) Giving rise to different DNA sequences resulting in the origin of new species including humans. Major changes in climate and environment can also lead to catastrophism and extinction of species on Earth; DNA, the most important molecule of heredity, concurrently developed and coded with the development of the cell is evidenced by the fact of matching DNA finger prints of parents and children and some planets occupy similar meridians besides the he number of DNA entries required to differentiate one from the other being also equal to the number of planetary configurations - all these factors have been explained in detail in the body of the paper as the factors in formulating 'Advanced Panspermia Hypothesis of Origin of Life and Hypothesis of Evolution'.

\subsection{Current State of Research}

There are problems facing abiogenesis like the problems that the cell architecture is not coded by DNA [1]. And that 
even the simplest cell has a sophisticated control system which drives the cell's life cycle [2]. This problem is totally dormant in the panspermia hypothesis revived by Crick and Orgel [3]. Hoyle and Wickramasinghe [4] confirmed that life could have come from the cosmos and further research and evidences showed that bacteria and even higher organisms could have survived a journey through space. Molecular biology, and at intricate level cell Biology and genetic research has confirmed that life is far more complex than thought. Panspermia, therefore, is becoming a serious alternative to the theory of evolution. For the first time, in this paper, the importance of Geological Time Scale is utilised in arriving at the role of climatic changes at regular intervals in the origin and evolution of species.

Since it is doubtful that Darwinian evolution was responsible for the generation of complex organisms which needed thousands of beneficial mutations and also not able to explain the origin of life and evolution in such intricate organs like human brain and circadian motions of the eye, it was proposed here that strong and 'Advanced Panspermia' could be responsible for further development. Yet, another point to totally dispute is the circumstance that during the Cambrian explosion, around 540 million years ago and particularly during the 20-million-year period all existing phyla belonging to the animal kingdom suddenly and simultaneously appeared as vertebrates of complex nature in total contra distinction to 'Darwin's Theory of Evolution and Natural Selection' regarding evolution of species over efflux of geological time Periods and Eras.

\subsection{Stars}

Milky Way galaxy revealed that the human body consists of ninety-seven percent of stardust. More than 97 percent of the mass of a human body is made up of carbon, hydrogen, nitrogen, oxygen, phosphorus and sulphur "CHNOPS" the six most common elements of life on Earth. This establishes an undeniable fact that most of the essential elements of life are made in stars. Many of the atoms which make up our body were created sometime in the distant past inside of stars, and those atoms might have plausibly made long journeys in the form of star dust from those ancient stars to us. What are stars made of and what are they finally becoming are all celestial questions. Jennifer Johnson, an astronomer concluded that we are made of star dust since most atoms in our bodies were forged in stars, and the cosmic processes are as varied as the atoms they produce. Under abiotic conditions, these are synthesised into all life building blocks spontaneously in space leading to membrane-bound proto-cells to finally pre-programmed living cells of different species [5]. "The proposition that space dust collisions could propel organisms over enormous distances between planets raises some exciting prospects of how life and the atmospheres of planets originated. The streaming of fast space dust is found throughout planetary systems and could be a common factor in proliferating life" [6]. Such an event could enable bacteria and other forms of life can easily make their way from one planet in the solar system to another and perhaps beyond to interstellar space. The average adult human is made up of 7 octillion atoms, and most of them are hydrogen - the most common element in the Universe, produced by the Big Bang about 14 billion years ago. The Sun, centre of the solar system, has $75 \%$ Hydrogen and $25 \%$ Helium. The central strands of the human DNA are of Hydrogen. Thus, a fundamental harmony exists between the universe and the individual. So, the human being is microcosm of the macrocosm, the universe.

\section{Semi Harmonic Climate Models and Geological Time Scale}

Climate and environment have a great role in the evolution and extinction of species. Climate and environment on earth are guided by the planets of the Solar System including the earth itself. Earth's elliptical orbit around the Sun changes in a cycle of around 100000 years in two respects. Given the fact that lower than 100,000-year cycles are comparatively weak particularly in the changes of climate and environment in tune with Milankovitch Climate Cycles [7]. Kepler also conjectured that celestial harmonics could permeate the entire solar system including the Earth's climate (Kepler, $1601,1606,1619)$. The solar system works as a resonator characterized by a specific harmonic planetary structure that synchronizes also the Sun's activity (modulation of the $\approx$ 11-year Solar cycle) and the Earth's climate [8].

In full conformity with Kepler's vision about the existence of a harmony, innumerable empirical studies have proved that a complex synchronized planetary harmonic order characterize not only the solar planetary system but also the Sun's activity and the Earth's climate of the world. This in turn aided the origin and evolution of each of the species with each great change in climate taking place at regular harmonic intervals of 'Periods' of Geological Time Scale.

Two-body harmonic orbit resonances of the 6 neighboured planet pair systems of solar system, excluding the outer planets beyond the orbit of Saturn, arise from the fact that one frequency or the other of the myriad frequencies match with every one of the planets and give rise to different sequences of DNA, thus cause of origin of different totipotent cells of species. Orbit resonances of the 6 neighboured planet pair systems of solar system are as follows (Table 1). The outer planets which are post Kepler are excluded because of their great distances from the Sun with weak Solar Energy.

Table 1. Two-body harmonic orbit resonances of the 6 neighboured planet pair systems of solar system.

\begin{tabular}{lllll}
\hline Number & Planet & Orbital Period (Years) & Neighboured Planet Pair Systems & Harmonic Planet Pair Orbit Resonance \\
\hline 1 & Mercury & 0.241 & Mercury - Venus & $(5: 2)$ \\
2 & Venus & 0.615 & Venus - Earth & $(5: 3)$ \\
\hline
\end{tabular}




\begin{tabular}{lllll}
\hline Number & Planet & Orbital Period (Years) & Neighboured Planet Pair Systems & Harmonic Planet Pair Orbit Resonance \\
\hline 3 & Earth & 1.000 & Earth - Mars & $(2: 1)$ \\
4 & Mars & 1.881 & Mars - Jupiter & $(5: 2)$ \\
5 & Jupiter & 11.862 & Jupiter - Saturn & $(5: 2)$ \\
\hline
\end{tabular}

Amongst these two-body harmonic orbit resonances, Mercury-Venus pair and Jupiter-Saturn pair have similar values of $5: 2$, but the latter is considered the dominant harmonic orbit resonance since Jupiter's atmosphere is similar to that of the Earth besides Jupiter is by far the most massive of all planets with maximum magnetic field (gauss) at the equator and Saturn trails it. Though the harmonic orbit resonances of planets outside the orbit of Saturn are greater than that of Jupiter-Saturn, they are not considered since the incident sunlight on Uranus, Neptune and Pluto is insignificant; ipso facto the reflected sunlight is negligible. A full planetary alignment on one side of the Sun, will take place only once in over 1.6 million years due to the large orbits of outer planets. A full alignment with planets on either side of the sun is possible once in approximately every 516 years [9].

Kepler's Successive Pairs of Planetary Synchronisation Structure of the Solar System and Semi Harmonic Climate Models based are based on the dominant ratio of 5:2 Jupiter-Saturn pair. While Jupiter makes 5 revolutions, Saturn makes only 2 revolutions and thus meet approximately at their same location positions of the zodiac once every 60 years; while doing so, Jupiter crosses Saturn twice, once every 20 years resulting in three conjunctions (Figure 1).

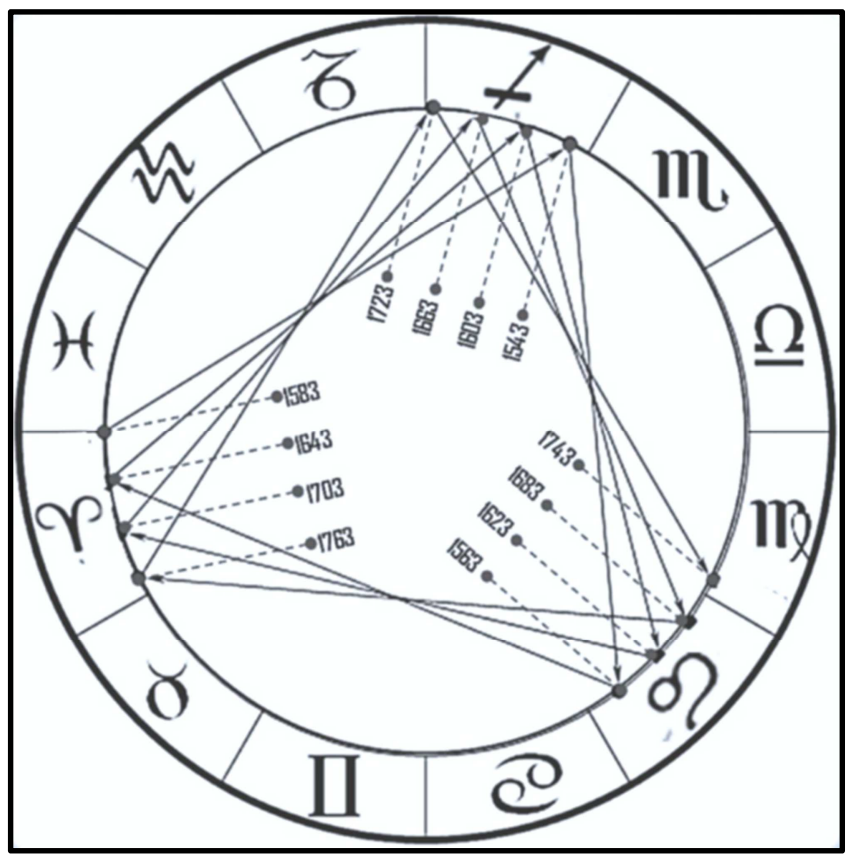

Figure 1. Kepler's trigon junctions. Three conjunctions (a trigon) of Jupiter and Saturn as an Equilateral Triangle.

\subsection{Major Mass Extinctions of Life and Climate Change}

Throughout the Earth's history of 4.6-billion-years, the breaking up and formation of supercontinents appear to be cyclical. During the 4.6 billion years of history of the Earth, there have been five known major mass extinctions that wiped out an overwhelming majority of all species living at that time. All the following five major mass extinction events occurred are of varied sizes and are due to several causes chief of which are climate change, geologic catastrophise such as the breaking up and formation of supercontinents. It is observed that all of them wiped out and devastated the biodiversity found on Earth at the times when they happened from 440 million years to 200 million years ago, at multiples of 60 million years.

i. The Ordovician Mass Extinction during the Ordovician Period of the Palaeozoic Era (about 440 million years ago) and the possible cause could be Continental Drift and subsequent climate change;

ii. The Devonian Mass Extinction during the Devonian Period of the Palaeozoic Era (about 375 million years ago) possibly due to lack of oxygen in the oceans, quick cooling of air temperatures, possibly volcanic eruptions and/or meteor strikes;

iii. The Permian Mass Extinction during the Permian Period of the Palaeozoic Era (about 250 million years ago), reasons unknown, Lack of oxygen in the oceans, quick cooling of air temperatures, possibly volcanic eruptions and/or meteor strikes;

iv. The Triassic-Jurassic Mass Extinction during at the end of the Triassic Period of the Mesozoic Era (about 200 million years ago), Major volcanic activity with basalt flooding, global climate change, and changing $\mathrm{pH}$ and sea levels of the oceans; and

v. The Cretaceous-Tertiary (or the K-T) Mass Extinction at the end of the Cretaceous Period of the Mesozoic Era (about 65 million years ago) possible due to extreme impact of asteroid or meteor.

With the onset of new 'Eons' and 'Eras' and 'Periods', coincidentally there is an emergence of a set of new species due to major congenial climate and environment conditions while extinction of species occurred whenever the climate and environment conditions of catastrophism resulted. Throughout the Earth's history of 4.6-billion-year, the breaking up and formation of supercontinents appear to be cyclical.

In all, five extinctions have occurred so far, the last one being Dinosaur mass extinction about 65 million years ago, which marks the end of the Cretaceous period of Mesozoic Era and the beginning of the Paleogene Period of Cenozoic Era. Interestingly enough, Mesozoic Era is about 180 million years, marks the beginning of the breakup of Pangaea. Continental Drift has affected the world's geographical positions and the world's climates and the evolution of animals besides the horrendous effects like Earthquakes and Tsunamis.

Kepler (cf. Ptolemy, II century), Kepler (1601) contended 
that the Earth's climate system could be partially synchronized to multiple planetary harmonics (Kepler, 1601, 1606). While doing so, Kepler identified the main long scale harmonics like a 20 -year oscillation and a 60 -year oscillation. These oscillations were suggested by the conjunctions of Jupiter and Saturn corresponding to historical chronological considerations (Kepler, 1606; Ma'Sar, IX century). A 60year cycle was included in Chinese and Indian traditional calendars since times antiquity probably because these cycles also reflected in the monsoon cycles [10]. Hence, multiples of about 20 million years, 60 million years and 600 million years for Periods, Eras and Eons respectively are recognisable. Interestingly enough, Periods, Eras and Eons in the Geological Time Scale, are multiples of about 20 million years', 60 million years' and 600 million years' duration respectively which are shown in the Geological Time Scale marked in Red colour (Figure 2) for brevity.

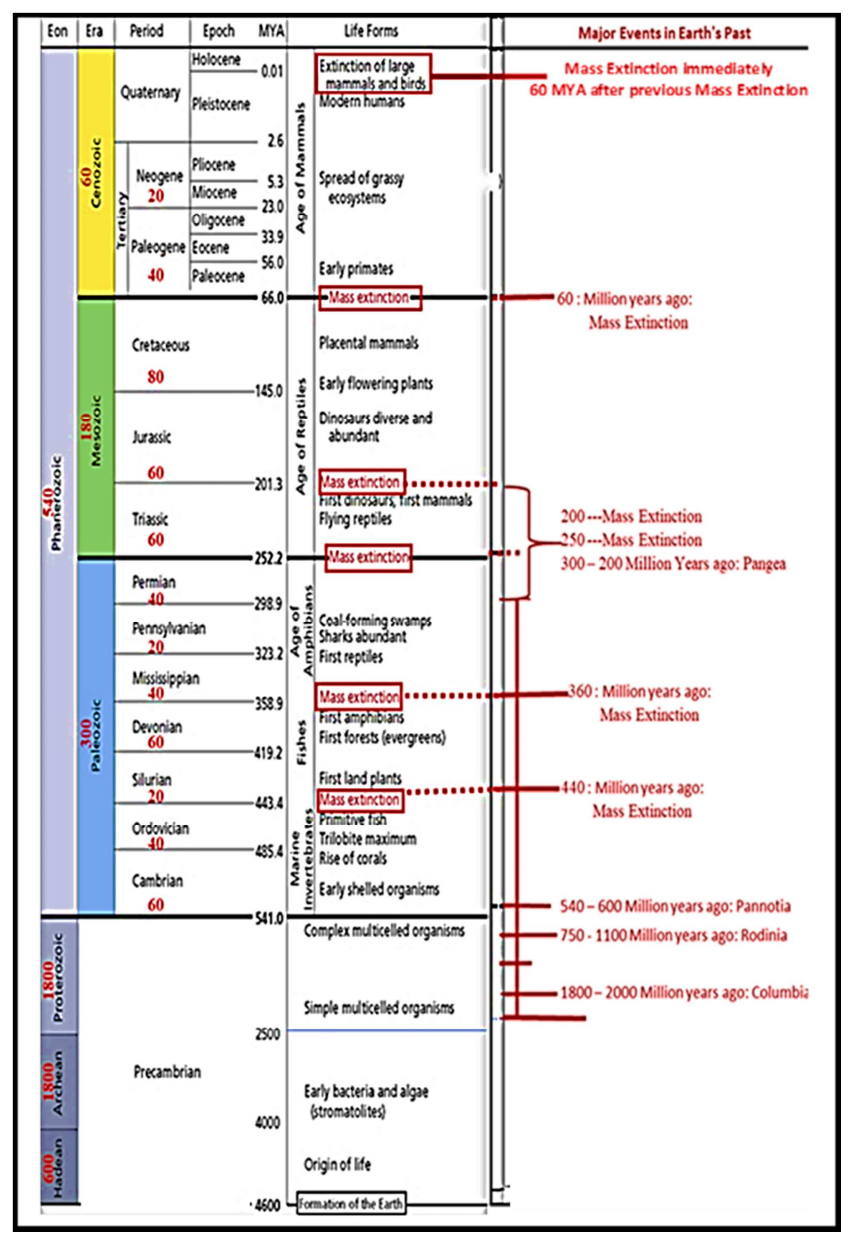

Figure 2. Geological Time Scale.

\subsection{Cambrian Explosion}

The Cambrian explosion was an event around 541 million years ago in the Cambrian period when most major animal phyla suddenly appeared. It lasted for about 20-million-year period and resulted in the divergence of animals as vertebrates. Because of the relatively short time over which this diversity of forms appears not in sequence but simultaneously in more complex forms. Before the Cambrian Period marks an important point in the history of life on Earth most organisms were simple, composed of individual cells and all present animal phyla appeared during this period. A glimpse of the building process of highly complex new organisms wherein every cell in the body specifies its position and type clearly reveals that advanced panspermia alone could be responsible for the whole mechanism of development.

\section{Concurrent Origin of Genetic Code and Origin of Life}

The question whether there is any scientific connection either direct or indirect between what goes on in the heavens for "Origin of Life" and effects perceived on Earth in the form of "Evolution of Life" is no longer a problem. As space physics, has moved on a great deal and a great deal is known about solar wind and how the Sun's magnetic field encompasses interstellar space and our system and beyond. This combination of solar wind and the magnetosphere of the Sun is known as the Interplanetary Magnetic Field (IMF) and extends far into interstellar space, engulfing the whole solar system. Many planets have large magnetic fields of their own, as does our Earth. Venus has no magnetic field while the Moon and Mars are patchy, both are considered to be largely non-magnetic. However, even non-magnetic planets cause disturbances in the IMF.

Majority of the theories on the origin of the genetic code have assumed that the genetic code emerged out of direct chemical interactions between amino acids and their possible codons or anticodons due to asymmetric magnetic force [11, 12]. Totipotent cell together with its DNA, at various stages of its development under abiotic conditions, picks up different magnetic signals from out of the solar symphony and the DNA is thus pre-programmed and finally lead to living cells. The magnetic memories may be evoked later in evolution when similar magnetic signals are repeated. Different magnetic signals from out of the solar symphony at different times of synchronisation of successive planetary pairs of Solar System give rise to multiple DNA sequences of multiple species of myriad frequencies wherein the successive pairs of planets plausibly represent pairs of codon and amino acids circles. Earth's magnetosphere contains a wide range of frequencies, one of which is connected to the Moon. Similarly, other planets show myriad bands of frequencies of their magnetic fields. Such of those frequencies in the magnetosphere of a planet that are close to the tidal frequencies of the other planets will remain in step with one another. Thus, each planet will have one magnetic frequency associated with one planet, another with some other planet and so on. Successive Pairs of Planetary Synchronisation Structure of the Solar System will remain in step with one another and their electromagnetic force help the origin of different genetic codes of different species. Mechanism of 'Origin of Life' fortifies the mechanism for 'Evolution'. 
Female Menstruation cycle is an important physiological process in the Reproduction System. The entire process is orchestrated by the electromagnetic forces of the Sun, the Earth and the Moon. The Lunar Phases match one to one with those of the Human Female menstrual phases both in terms of their duration and functions. This is an essential step in fortifying the conclusion that evolution of species on the Earth is the result of the solar symphony on the principle "as above so below". It is therefore reasonable to hold that the same mechanism (solar symphony) plays its part in the origin of life as well as in evolution of life.

\section{DNA: Most Famous Molecule of Heredity}

Deoxyribonucleic acid (DNA) is a molecule that contains the biological instructions that make each species unique. DNA, along with the instructions it contains, is passed from adult organisms to their offspring during reproduction. DNA is the most famous molecule of heredity. The foetus's neural network serves as antenna. It tunes into fluctuations in Earth's magnetic field. The imprinting of the neural antennae depends on genetic heredity. This gives us our basic congenital personality. If a certain planet in a parent's planetary configuration at his birth time was placed in a particular zodiacal sign, the child also is born with a similar planetary placement. The percentage of similarities of planets' places depends on the number of same planets' occupation in similar signs similar to the number of bands matching in DNA fingerprinting [13]. French statistician and scientist Michel Gauquelin's work, in which human biological clocks keep time with the planets. Gauquelin's studies, which showed striking planetary similarities in the birth charts of parents and their children, comprise the strongest scientific evidence in support of 'Advanced Panspermia Hypothesis of Origin of life' to date.

Gauquelin's Planetary Heredity Tests of planetary links between parents and children seemed positive. He demonstrates that we were born under similar cosmic conditions as prevailed at the birth of our parents and graphs a relationship between human heredity and the star. Every family member is linked with each other from certain planets. Gauquelin's studies showed striking planetary similarities in the planetary configurations of parents and their children for their birth times [14]. This fact comprises the strongest scientific evidence in support of "Advanced Panspermia Hypothesis of Origin of Life and Evolution of Life on Earth".

\subsection{Planets' Configurations and DNA Entries}

What is stated above is the correct view of the matter is evidenced by the fact that the number of DNA entries required $\left(3 \times 10^{6}\right)$ and the number Planets' configurations $12^{6}$ i.e., $2,985,984 \approx 3 \times 10^{6}$ to differentiate one person from the other is the same in both the cases [15].

Extra-terrestrial astral influence affects earth's geomagnetic fields, which in turn affect human foetal development. That the above concept of Moon and Magnetism and orbital revolutions are responsible in orchestrating female menstrual ovulation cycles, fertilisation and finally heralding child birth in the symphony of life is the correct view of the matter is evidenced by the fact that the number of planets' configurations and the number of DNA entries required to differentiate two individuals from one person to the other are found to be identical.

Human genome comprises of over 3 billion i.e., $3 \times 10^{9}$ of DNA base pairs of which $99.9 \%$ are identical from one person to the other. The. residual $0.1 \%$ i.e., $3 \times 10^{6}$ allows us to differentiate one person to the other, in terms of appearance, character and also allow effective human identification through DNA typing or DNA profiling. With the world's human population estimated to be only 7.1 billion i.e., $7.1 \times 10^{9}$, it is therefore extremely unlikely for one to share the same profile as someone else, unless one is an identical twin.

\subsection{Planets' Configurations}

The fact that striking planetary similarities are observed in the planetary configurations of parents and their children for their birth times and that the value of DNA entries being equal to the number of planetary configurations with individual degrees of freedom of planets comprise the strongest scientific evidence for the 'Advanced Panspermia Hypothesis of Origin of Life', and the 'Hypothesis of Evolution of all Life on Earth'.

\subsection{Human Female Menstrual Cycle}

Human Female Menstrual Cycle exhibits a close relationship with the Phases of the Moon during Lunar Month of 29 Days in terms of their duration as well as their functions. This relationship is correlated and presented as follows for brevity (Table 2). Specific moon phase energies, when they magnify phases of menstrual cycle, can be freed up and made available for use if we choose to work with them. Lunar Magnetism orchestrates Human Female Menstrual Cycles. Lunar Phases match one to one with human female menstrual phases match one to one in terms of duration and nature of functions comprise the strongest scientific evidence of Panspermia Hypothesis of Origin of Life and Hypothesis of Evolution of all Life on Earth [16].

Table 2. Correlation of Human Female Menstrual Phases and Phases of the Moon of their duration as well as their functions.

\begin{tabular}{lllll}
\hline $\begin{array}{l}\text { Sl. } \\
\text { No. }\end{array}$ & $\begin{array}{l}\text { Phases of Human } \\
\text { Cycle }\end{array}$ & $\begin{array}{l}\text { Relation Phases of } \\
\text { Moon to Phases of } \\
\text { Menstrual Cycle }\end{array}$ & $\begin{array}{l}\text { Duration of } \\
\text { Phases in Both } \\
\text { the cases }\end{array}$ & $\begin{array}{l}\text { Comparison of Nature of Function and Duration of Phases in Both } \\
\text { the cases }\end{array}$ \\
\hline 1 & Menstrual Phase & $\begin{array}{l}\text { Beginning of New Moon } \\
\text { to First Ascent }\end{array}$ & Day 1 to day 5 & $\begin{array}{l}\text { Discharge of black/brown blood similar to } 5 \text { dark nights, the uterus } \\
\text { sheds the lining (endometrium) built up in the previous menstrual cycle }\end{array}$ \\
\hline
\end{tabular}




\begin{tabular}{|c|c|c|c|c|}
\hline $\begin{array}{l}\text { Sl. } \\
\text { No. }\end{array}$ & $\begin{array}{l}\text { Phases of Human } \\
\text { Female Menstrual } \\
\text { Cycle }\end{array}$ & $\begin{array}{l}\text { Relation Phases of } \\
\text { Moon to Phases of } \\
\text { Menstrual Cycle }\end{array}$ & $\begin{array}{l}\text { Duration of } \\
\text { Phases in Both } \\
\text { the cases }\end{array}$ & $\begin{array}{l}\text { Comparison of Nature of Function and Duration of Phases in Both } \\
\text { the cases }\end{array}$ \\
\hline & & & & $\begin{array}{l}\text { as the Moon sheds its darkness. Hormones are at their lowest similar to } \\
\text { lowest illuminated Lunar disc. }\end{array}$ \\
\hline 2 & Follicular Phase & $\begin{array}{l}\text { New Moon to Full Moon } \\
\text { (Waxing Moon) }\end{array}$ & Day 1 to day 14 & $\begin{array}{l}\text { Uterine lining (endometrium) is regrown like the waxing moon. } \\
\text { Oestrogen levels increase during } 14 \text { days from New Moon. }\end{array}$ \\
\hline 3 & Ovulation Phase & New Moon & Day 15 & $\begin{array}{l}\text { Different levels of hormones are released and release of fully } \\
\text { developed egg like the Full Moon in shape and size. Oestrogen levels } \\
\text { peak at the Full Moon on the } 15^{\text {th }} \text { day. }\end{array}$ \\
\hline 4 & Luteal Phase & $\begin{array}{l}\text { End of Full Moon to } \\
\text { beginning of New Moon } \\
\text { (Waning Moon) }\end{array}$ & $\begin{array}{l}\text { Day } 16 \text { to day } \\
29\end{array}$ & $\begin{array}{l}\text { If the egg is not fertilised then the menstrual cycle continues for } \\
\text { another } 14-15 \text { days from day } 16 \text { to day } 29 \text {. Decline in release of levels } \\
\text { of hormones and endometrium begin to die and shed; both the } \\
\text { phenomena are similar to. } \\
\text { Waning Moon. }\end{array}$ \\
\hline
\end{tabular}

\section{5. "Advanced Panspermia Hypothesis of Origin of Life"}

The hypothesis of advanced panspermia is the most plausible mechanism of origin of fully self-contained pre-programmed totipotent cells in space. All the necessary constituents that go into the making of life building blocks are available as readymade kits in space [17]. Methane, Ammonia, Hydrogen and water available in plenty in space are synthesized through electrical discharge triggering lightning into Amino acids. Similarly, spontaneous formation occurred of lipids, Carbohydrates. Amino Acids, formation of Proteins - RNA Systems, Nucleotides and evolution of DNA and other enzymes under abiotic conditions leading to self-replicating enclosed membrane bound proto-cells and finally resulting in living totipotent cells during the early days of formation of solar system when lightning is plausible.

First pre-programmed Totipotent cells formed under abiotic conditions in space were brought by star dust from space to Earth for evolution and later, pre-programmed Totipotent cells of multicellular life was introduced on the Earth for evolution, when the Earth had stabilised and had enough oxygen. Successive pairs of planetary synchronization structure of the Solar System plausibly caused the origin of different DNA sequences and new species where revolution periods of the two successive planets of each pair plausibly signify the two concentric circles, one of codons and the other corresponding to amino acids. Programming provides the needed instructions for what the cell is required to do. The cell is required to make these programming choices before any processing such as to come alive, stay alive and reproduce. No living physical organism comes into existence until after an incredible amount of programming and processing produces it. The totipotent cell at different stages of its development picks up different magnetic signals from out of the solar symphony and the DNA is thus preprogramed. These pre-programmed magnetic memories may be evoked later in evolution when similar magnetic signals are repeated. There is no fundamental difference between a living organism and lifeless matter and the complex combination of manifestations and properties, genetic characteristics, of life must have arisen in the process of the origin of the cell as a complete unit (Totipotent cell) spontaneously and not after the organism has come to life and the organism responds to the instructions after coming to life. The electromagnetic force of successive pairs of planetary synchronization structure of the solar system give rise to multiple DNA sequences in the Totipotent cells of multiple species including humans. The first cell (Prokaryotic), a preprogramed, full-fledged and self-contained one and need nothing to manufacture here on Earth, mingled with the star dust and propelled at supersonic velocities throughout planetary systems including on the Mother Earth for evolution.

\section{ADVANCED PANSPERMIA HYPOTHESIS OF ORIGIN OF LIFE}

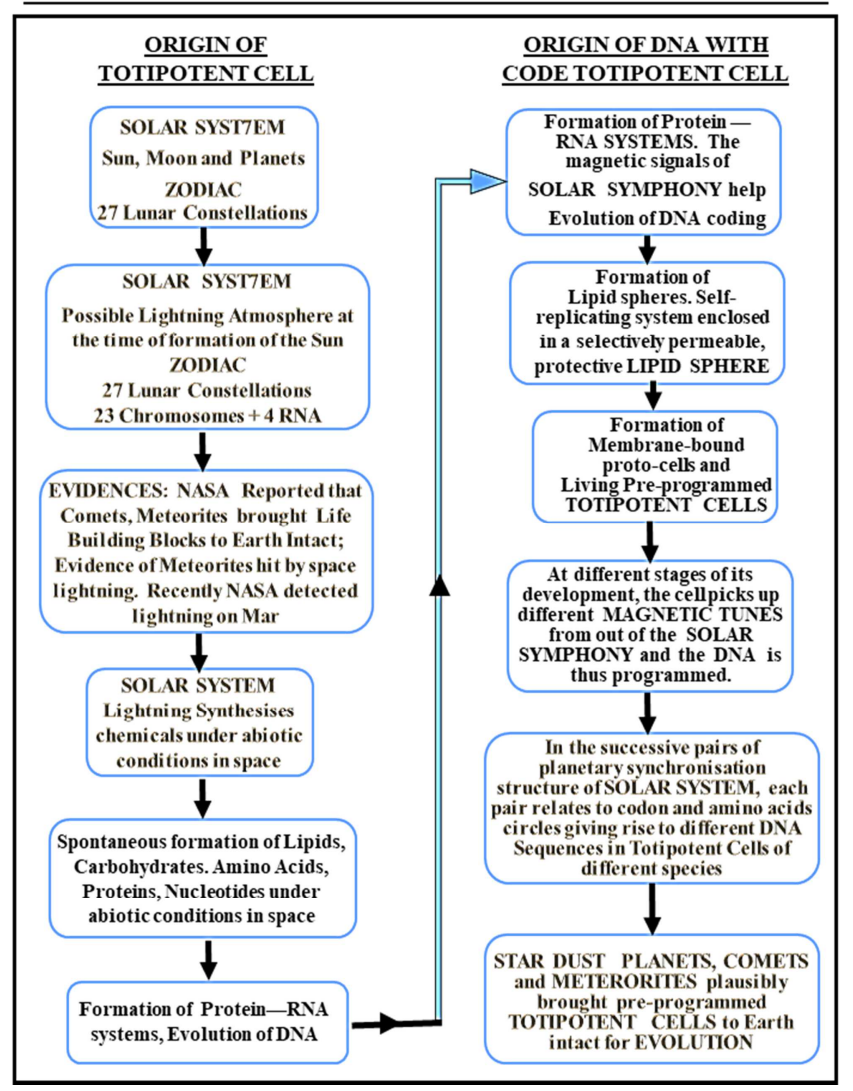

Figure 3. Flow Diagram of Advanced Panspermia Hypothesis of Origin of Life". 
Thus, life came on Earth 3.8 billion years ago during the Hadean Eon, simple totipotent multicell organisms about 2.0 billion years ago during early Proterozoic eon and later totipotent complex multicell organisms spontaneously arrived on the Earth at the far end of Proterozoic eon and at the beginning of Cambrian Explosion. The origin of life and origin of DNA is much closer. The flow diagram of "Advanced Panspermia Hypothesis of Origin of Life" is self-explanatory (Figure 3).

\subsection{Evolution: 'Darwin's Theory of Evolution and Natural Selection,}

Darwin's Theory of Evolution [18] is the widely-held notion that all life, from mammals to single celled organisms, is related and has descended from a common ancestor and that new species arise gradually with modification from pre-existing species; the result of competition among species is extinction of the less fit. Darwin, familiar with the findings of geologists that the Earth was far older than previously thought, gave him enough strength for his proposition of 'natural selection.' The theory states that all species of organisms arise and develop through the natural selection of small, inherited variations that increase the individual's ability to compete, survive, and reproduce. The most contentious evolutionary issue is the origin of humans on this earth.

All living things have the same genetic substance DNA. Only the sequence of the four nucleotides is different. Humans are different from dogs only because their DNA is composed of a different ordering of nucleotide rungs in the double-helix ladder. Evolution is just as substantiated as the theory of Electromagnetism. The whole universe is full of vibration. Our problem is with the Darwinian story-telling about the past. Until now, Darwin's Theory of Evolution is held prime for want of a better explanation than evolution and natural selection, despite some stout arguments against this theory. Darwin himself refuted his own theory in the case of humans born with irreducible complex system. "If someone could really demonstrate a better explanation than evolution and natural selection, [that person] would be the new Darwin," ---- Richmond [19].

\subsection{Examination of 'Darwin's Theory of Evolution and Natural Selection'}

"There is no fundamental difference between a living organism and lifeless matter. The complex combination of manifestations and properties so characteristic of life must have arisen in the process of the evolution of matter." --Oparin, A (1938) [20]. This concept is also totally ridiculous like Darwin's Theory. The complex combination of manifestations and properties so characteristic of life must have arisen in the process of the origin of a cell and not in the process of evolution of matter. Similarly, on a keen examination of Darwin's Theory of Evolution, it is evident that 'Natural Selection' does not produce anything.
It can only "select" from possible biological variations of what is produced. Darwin's Theory of Evolution can only "select" from variations that are genetically possible and which have survival value. Natural selection is a passive process. These biological variations are possible in nature, not natural selection. The complex combination of manifestations and properties so characteristic (genetic trait) of life must arise not in the process of the evolution of matter but at the time of origin of life and not later. The genetic information for a certain trait or characteristic is already present in the DNA (or genetic code) of a species and the environment triggers the capability of putting that information there so that the species would develop that particular trait or characteristic. The environment can only work with the genetic potential already existing in species in contradistinction to Darwin's Theory of Evolution and Natural Selection. If the genetic information for a certain trait or characteristic is not already there in the DNA (or genetic code) of a species, then there is nothing in the environment that can put that information there so that the species would develop that particular trait or characteristic. The environment can only work with the genetic potential already existing in species, but what the environment cannot do is increase that genetic potential. DNA is a molecule that contains the biological instructions that make each species unique. With enough time and with enough mutations entirely new genes for new traits will not be produced, will not be preserved by natural selection and will not lead to the evolution of new biological kinds. There is no evidence that this can happen as it is impossible to change a biology book into a book on astronomy by accidentally changing the sequence of the letters in the DNA Code. There is no common ancestor either. This clearly suggests that Darwin's Theory of Evolution and Natural Selection is ridiculous.

Since the dawn of human history, humans have sought to discover the forces by which the planets influence life on Earth. Recent researches in magnetobiology showed that a wide variety of organisms including humans responded to changes in Earth's magnetic field [21]. Eysenck and Nias suggest that solar disturbances and their particle emissions are the most probable link between biological and extra-terrestrial events [22]. Planets raise tides in the gases of the Sun, creating sunspots and their particle emissions, which then travel across interplanetary space and strike Earth's magnetosphere. These planetary magnetic signals are then perceived by the neural network of the foetus inside the mother's womb, heralding the child's birth. Earth's magnetosphere contains a wide range of frequencies, one of which is connected to the Moon. Resonance can happen when the frequency of Moon closely matches a frequency already present within Earth's magnetic field. It then becomes phase-locked. The foetus is genetically predisposed to hear specific planetary signals, much as a radio is resonantly tuned to receive a particular station [23]. 


\section{Human Female Menstruation Cycle and Child Birth}

Human Female Menstruation is an important physiological process in the Reproduction System. Menstruation occurs on a monthly cycle throughout female reproductive life. A better understanding of the rhythmicity of phases of moon and its electromagnetic energy influences orchestrated by its revolution helps us, develop a better and more harmonious relationship with it and appreciate the rhythmicity of female human menstrual phases which are entirely in harmony with it in the symphony of life in her reproduction system heralding child birth. "It's called the lunar effect, and, as far as births are concerned, the primary explanation for the effect focuses on the moon's gravitational pull. It basically states that much the way the moon's gravity controls the tides, it can control a woman's body. The human body is 80 percent water, after all. This argument put forth here is based on single-link theory which are simple models which can be easily disproved. This approach ignores the possibility of multi-link theories. Gravity can't possibly work because of the weak tidal tug of the planets Given that both menstruation and ovulation roughly follow a lunar cycle -- occurring on a monthly basis -- it doesn't seem too far off to think that the moon could have a say in childbirth as well [24]. Mechanism involved in the "Foetus Development and Child Birth" enables us to have a better understanding of "Evolution of Life on Earth"

\subsection{New Moon and Menstruation}

Month after month, the moon cycle mirrors a woman's menstrual cycle, which coincidentally has an average length of 29.5 days as well. Similar to the moon cycle, a woman's menstrual cycle changes from the menstruation of new growth, to the ovulation of full power and blossom, to the pre-menstrual phase of harvest and degeneration, and back to menstruation of renewal again. Menstruation occurs on a monthly cycle throughout female reproductive life. Women's menstrual cycles begin more often during a New Moon. Studies found a synchronous relationship between the menstrual cycles and lunar rhythm. Among 826 females with a normal menstrual cycle, aged between 16 and 25 years, more than 28 percent of all menstruations occurred around the New Moon. The proportion of menstruations at other times during the lunar month ranged between 8.5 and 12.6 percent [25].

\subsection{Full Moon and Delivery of Child}

Pregnant women spontaneously deliver more often during a Full Moon. The study that examined all full-term spontaneous deliveries (without mechanical or drug intervention) at the Civil Hospital, Fano) during a two-year period found a significant relationship in women who had one or more previous pregnancies and their deliveries clustered around the Full Moon [26]. Other research analysis conducted a) During 1996, Joshi et al examined the relationship between the lunar position and spontaneous deliveries, especially in relation to the presence of a full moon [27]. b) A retrospective analysis of 1248 spontaneous full-term deliveries in a three-year period (36 lunar months) was done at the Department of Obstetrics and Gynaecology, Civil Hospital, Fanon (Marche, Italy). Both these studies showed a connection between spontaneous full-term deliveries and the lunar month.

\subsection{Variable Menstrual Periods: Natural Cure}

Usually, the regular menstrual period lasts a lunar month of 29 days. Not all women do have regular periods. Regular monthly period in females is plausibly affected due to changes in life styles resulting in any mismatch between the internal biological menstrual cycle and the regular lunar rhythmicity and Moon Light intensity. It is a common notion that the natural cycle of women who live and sleep out in the open away from artificial light is to ovulate on the full moon and menstruate on the new moon. But this may not be the case for most modern women whose natural rhythms are largely affected by artificial light. Some women may menstruate on the new moon, some on the crescent moon, some on the full moon, some on the waning moon, and others on the new moon. Before electricity and the light bulb were invented, shifts in the amount of light intensity at night during the moon's various phases could possibly have played a significant role in our menstrual cycle of Moon by affecting certain body processes impacted by light exposure, such as the production of the hormone, thus prompting tying a relationship of female menstrual cycle to the moon cycle.

An interesting 1990 study in the Journal 'Psychiatry Research' found that bright light at night can help regulate the menstrual cycle. In the study, women with abnormally long cycles of about 45 days who slept with a 100 -watt bulb turned on at night in their bedroom from Day 13 to Day 17 of their cycle successfully shortened their cycles to about 33 days. Light is the reflected Sunlight and the Sunlight itself is an electromagnetic wave of light and particles. This is the correct view of the matter is evidenced by the fact that Women with variable onset of menstrual periods, artificial illumination of the bedroom through the 14th to 17 th nights following the onset of menstruation resulted in the regularization of the period [28].

\section{Hypothesis of Evolution of Life on Earth}

The various life forms are nothing but the degrees of omnipresent manifestation of electromagnetism in the universe. The hypothesis of evolution proposed hereinafter shall henceforth be known as "New Hypothesis of Evolution". It is defined as follows:

The Sun, Moon, and planets telegraph their effects to us via magnetic signals Omnipresent throughout the universe, magnetism is known to affect the biological cycles of numerous creatures here on Earth, including humans. Multi-link theory proposed by Percy Seymour states the 
planets raise tides in the gases of the Sun, orchestrating sunspots and their particle emissions, which then travel across interplanetary space to strike Earth's magnetosphere [29]. The combination of solar wind and the magnetosphere of the Sun called Interplanetary Magnetic Field (IMF), in turn affects the earth's magnetic field. These planetary magnetic signals are then perceived by the neural network of the organisms heralding symphony of life. As has been said the totipotent cell at different stages of its development, picks up different magnetic signals from out of the solar symphony and the DNA is thus programed rather coded and that these pre-programmed magnetic memories may be evoked later in evolution when similar magnetic signals are repeated. "Schumann Resonance, the very pulse of the Earth $7.83 \mathrm{~Hz}$, the frequency of Earth's natural harmonics. Only the organism that which is genetically predisposed to hear specific planetary signals, much as a radio is resonantly tuned to receive a particular station would evolve. Earth's magnetosphere contains a wide range of frequencies. Different species harmonically tune to the earth's natural frequency and evolve. Pre-programmed Totipotent cells of different species arrive at different geological time periods over different geographical locations by selection from possible biological variations in nature or environment that are genetically possible and which have survival value.

\section{EVOLUTION OF LIFE ON EARTH}

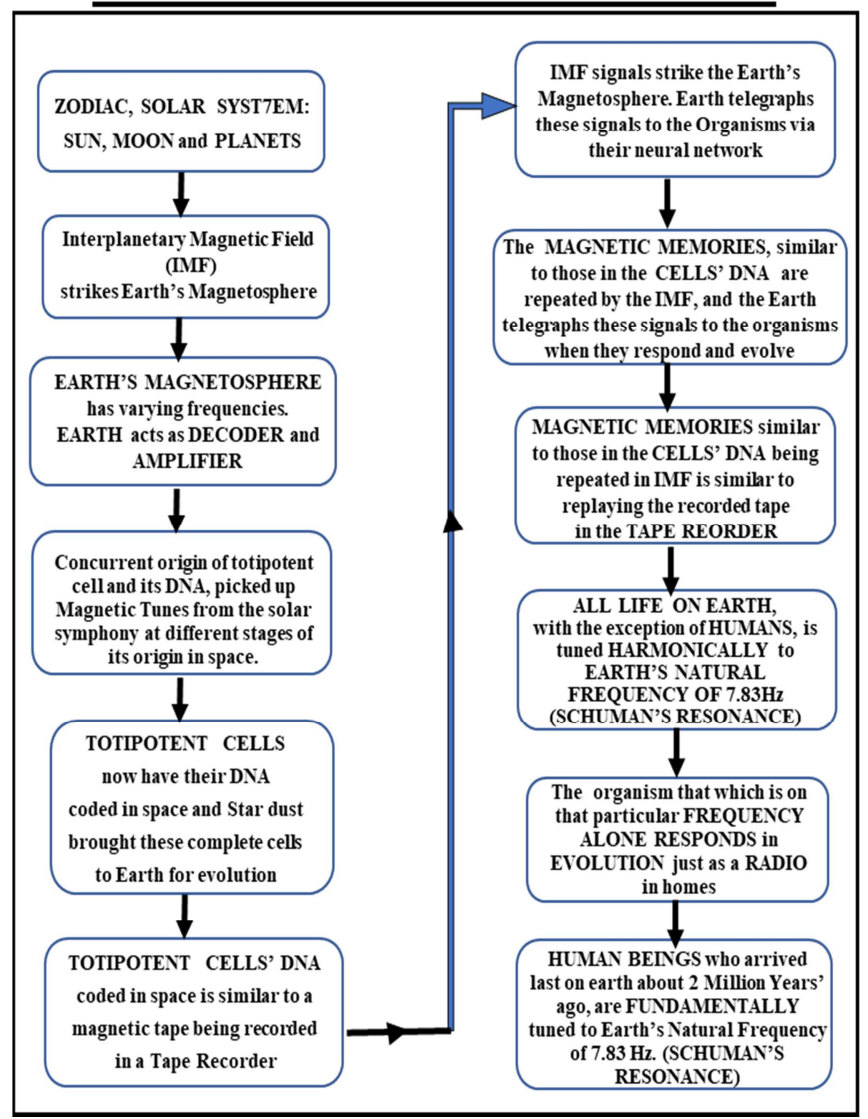

Figure 4. Flow diagram of "New Hypothesis of Evolution of Life on Earth".

Human beings, with irreducible complex organs, are tuned fundamentally to the natural harmonic frequency of the Earth, arrived last about 2 million years ago, and evolved all over the globe, thus, remain unique from rest of the species and are able to live in sweeping changes of climate and environment such as subfreezing temperatures of Antarctica as well as hot temperatures of Sahara Desert through the millennia. Human being is thus ultimate and unique in evolution. The mechanism involved in the birth of a child will be an invaluable guide for a clear understanding of what all is said above regarding evolution. So far, it is not known that there exists life on any inhabitable planet in other solar systems in the Universe, except on our Earth, Earth's magneto sheath acts as screen in filtering harmful UV and $\mathrm{X}$-rays from reaching the earth. This helps in the evolution of life of flora and fauna and therefore the Earth is aptly called "Mother Earth". The following is the flow diagram of "New Hypothesis of Evolution of Life on Earth" which is self-explanatory (Figure 4).

\section{Discussion}

Origin of cells and origin of DNA are simultaneous. Totipotent cells are formed in space under abiotic conditions without DNA Code. DNA of the Totipotent cell is similar to a Magnetic Tape. DNA of the Totipotent cell, at different stages of its development, picks up different magnetic signals from out of the solar symphony and the DNA is thus pre-programmed just as in a magnetic tape recorder and the cell emerges as living cell. The magnetic memories in the cell may be evoked later in evolution, when similar magnetic signals are repeated in Solar Symphony. This is similar to replaying the recorded tape in a recorder.

The solar system works as a resonator characterized by a specific harmonic planetary structure that synchronizes also the Sun's activity (modulation of the $\approx 11$-year Solar cycle) and the Earth's climate. Electromagnetic force of synchronisation of successive pairs of planets of Solar System plausibly relate to the following important aspects in 'Origin and Evolution'

The famous Miller-Urey experiment (1950) demonstrated abiogenesis, the process responsible for the development of living beings from non-living or abiotic matter could be synthesized through electrical discharge triggering lightning (Miller 1953) without the DNA code.

Lightning could cause chemical reaction under abiotic conditions in space. Many meteorites show evidence of having been hit by space lightning. Perhaps, this was in the early days of the solar system, when the sun had a thin atmosphere of gas and dust stretching far into the outer Solar System. Recently lightning was observed on Mars.

Many of the atoms which make up our body were created sometime in the distant past inside of stars, and those atoms might have plausibly made long journeys in the form of star dust from those ancient stars to us.

Astrobiology Division of NASA confirmed that life building blocks of DNA, Amino Acids, etc., are available in space as readymade kits and brought to Earth intact by 
Meteorites and comets. This finding leads us to conclude that lightning occurs in space also and could cause spontaneous formation of lipids, Carbohydrates. Amino Acids, formation of Proteins - RNA Systems, Nucleotides and evolution of DNA and other enzymes under abiotic conditions leading to self-replicating enclosed membrane bound proto-cells and finally resulting in living totipotent cells with DNA coded by picking up different magnetic signals from out of the solar symphony at different stages of its development.

Kepler's Successive Pairs of Planetary Synchronisation Structure of the Solar System: Two-body harmonic orbit resonances of the 6 neighboured planet pair systems of solar system arise from the fact that one frequency or the other of the myriad frequencies of planets match with every one of the planets and give rise to different sequences of DNA, thus cause of origin of different totipotent cells of species.

Space dust collisions could propel organisms over enormous distances between planets raises some exciting prospects of how life and the atmospheres of planets originated. The streaming of fast space dust is found throughout planetary systems and could be a common factor in proliferating life on Earth.

We are made of star dust since most atoms in our bodies were forged in stars, and the cosmic processes are as varied as the atoms they produce. Under abiotic conditions, these are synthesised into all life building blocks spontaneously in space leading to membrane-bound proto-cells to finally pre-programmed living cells of different species. Space dust collisions could propel organisms over enormous distances between planets raises some exciting prospects of how life and the atmospheres of planets originated. The streaming of fast space dust is found throughout planetary systems and could be a common factor in proliferating life on Earth.

So far, the only known inhabitable planet with life on it in the universe is our mother earth. Earth's Magneto-sheath is a protective layer which prevents harmful UV and X-rays from the sun from reaching the Earth; thus, helps evolution of flora and fauna on Earth.

All life on Earth adapt their biological rhythm so that it is synchronized with the Earth's revolutions. The Earth behaves like a gigantic electric circuit. Its electromagnetic field surrounds and protects all living things with a natural frequency pulsation of 7.83 hertz on average — the so-called "Schumann resonance," All life on Earth, except humans, is tuned harmonically to the natural harmonic frequency of the Earth, Human beings, with irreducible complex organs, are tuned fundamentally to the natural harmonic frequency of the Earth, arrived last about 2 million years ago, and evolved all over the globe, thus, remain unique from rest of the species and are able to live in sweeping changes of climate and environment such as subfreezing temperatures of Antarctica as well as hot temperatures of Sahara Desert through the millennia.

It is the natural resonant frequency of the planet and matches that of human consciousness at the optimal alpha brain wave state. Mother earth's frequency has matched that of the human brain's average alpha frequency in which the human brain operates.

Nobel laureate Luc Montagnier also used the $7 \mathrm{~Hz}$ to $8 \mathrm{~Hz}$ range of frequencies to successfully "teleport" DNA from one glass beaker to another.

Astronauts, when returned to Earth only after their stay for a very short time in space felt very weak, distressed and disorientated. The condition necessitated that they need to be carried in stretchers from the spacecraft. Consequently, NASA installed equipment in the spacecraft to generate Schumann waves artificially.

The target frequency of Earth resonance is the next harmonic member in the sequence is 13 cycles per second. This new harmonic member of 13 cycles per second may become our new base resonant frequency perhaps beginning in another 20 million years in a 'new geological period'. This frequency may trigger resonance with the new grid-matrix complex which plausibly signals the close of the present cycle of evolution and the beginning of the "New Age". Such spikes may result in new inventions or discoveries by more advanced humans.

Human beings are tuned fundamentally to the natural harmonic frequency of the Earth. Perhaps, this is on account of the fact that the Earth and its satellite, the Moon, rotate synchronously; hence, their orbital resonance is 1. Besides, these two are involved in the formation of Zygote and development of the foetus throughout the gestation period. Thus, even when the next harmonic member of earth's natural frequency is 13, human beings still tune fundamentally to the new natural harmonic frequency member of 13 of the Earth.

DNA is the most famous molecule of heredity. The following two important findings comprise the strongest scientific evidence in support of "Advanced Panspermia Hypothesis of Origin of life" and "Evolution of Life on Earth" to date. Striking planetary similarities are found in the birth charts of parents and their children. The number of DNA entries required $\left(3 \times 10^{6}\right)$ and the number Planets' configurations $12^{6}$ i.e., $2,985,984 \approx 3 \times 10^{6}$ to differentiate one person from the other is found to be the same in both the cases.

"Advanced Panspermia Hypothesis of Origin of Life and Hypothesis of Evolution" is in contradistinction to 'Darwin's theory of Evolution' and 'Natural Selection'. Darwin's concept, as well as that of Oparin, that complex combination of manifestations and properties so characteristic of life must have arisen in the process of the origin of a cell and not in the process of evolution of matter is ridiculous. It is evident that 'Natural Selection' does not produce anything. It can only "select" from possible genetically possible variations of what is produced and which have survival value. The complex combination of manifestations and properties so characteristic (genetic trait) of life must arise at the time of origin of life and not later in the process of the evolution of matter. The genetic information for a certain trait or characteristic is already present in the DNA (or genetic code) of a species. The environment triggers the capability of putting that 
information there so that the species would develop that particular trait or characteristic. The environment can only work with the genetic potential already existing in species, but cannot increase that genetic potential.

\section{Conclusions}

The following facts comprise the strongest scientific evidence of in support of "Advanced Panspermia Hypothesis of Origin of life" and "Evolution of Life on Earth" to date. This claim about these events allows us to observe, repeat and test in almost all similar cases and they also reveal that planetary mechanism is involved in both the Origin of life and Evolution.

i. Of all the four known forces of Physics, namely, Electromagnetism, Gravity, Strong and Weak Nuclear Energy, Electromagnetism is the criterion in Origin of Life and Evolution.

ii. Origin of cell and origin of DNA and code are concurrent.

iii. The cell is manufactured in space under abiotic conditions in the early days of solar system when lightning was present.

iv. Evidences of life buildings blocks brought to earth by meteorites and comets.

v. Female Human Menstrual Cycle exhibits a close relationship of its four phases with the four Phases of the Moon during Lunar Month of 29 Days in terms of their duration as well as their functions.

vi. Studies found a synchronous relationship between the menstrual cycles and lunar rhythm.

vii. Women who had one or more previous pregnancies and their deliveries clustered around the Full Moon.

viii. Regular monthly period in females is plausibly affected due to changes in life styles resulting in any mismatch between the internal biological menstrual cycle and the regular lunar rhythmicity and Moon Light intensity.

ix. Artificial illumination of the bedroom through the 14 th to 17 th nights following the onset of menstruation resulted in the regularization of the period.

x. The number of Planets' configurations and number of DNA entries required is found to be the same in differentiating one person from the other is the same in both the cases.

xi. Striking planetary similarities are found in the birth charts of parents and their children. Plants, animals and humans adapt their biological rhythm so that it is synchronized with the Earth's revolutions.

xii. The neural network of the foetus may be synchronizing and tuning its biological clock into Earth's and Moon's magnetic fluctuations.

xiii. Throughout the entire nine-month gestation period, the neural network of the foetus may be synchronizing and tuning its biological clock into Earth's magnetic fluctuations.

xiv. Our own resonances to the pre-programmed magnetic signals may be evolving along with changes in Earth's magnetic field.

$\mathrm{xv}$. All life on Earth is tuned to the natural harmonic Frequency of the Earth except human beings who are tuned fundamentally to the Earth's Natural Frequency of $7.83 \mathrm{~Hz}$ (Schuman's Resonance).

xvi. The signals arising out of the interaction of IMF and Earth's Magnetosphere are telegraphed to the organisms on Earth through their neural network for evolution and the organism which is on that particular frequency alone responds in evolution just as radio in home.

xvii. Major changes in climate at regular Geological Time Periods cause origin of new species and mass extinction of species.

xviii.Human physiology is constantly subject to cosmic influences. There is a perfect, one-to-one correlation between the cosmic bodies-sun, moon, planets, and stars-and human physiology; Our DNA knows its connectedness with everything in the universe including how to behave with the Sun, Moon, Planets and Stars and the changes of the seasons, climate and environment. The combination of solar wind and the magnetosphere of the Sun is known as the Interplanetary Magnetic Field (IMF) and extends far into interstellar space, engulfing the whole solar system. Interplanetary Magnetic Field (IMF) constantly impregnates the Earth's Magnetic Field. The origin of the Cell and DNA are simultaneous. The cell picks up magnetic signals of solar symphony at various stages of its development in space and thus its DNA is coded with all the genetic instructions and then spontaneously brought to Earth for evolution. When these similar magnetic memories are repeated in the IMF, which strikes earth's magnetosphere, the earth telegraphs these signals the organisms via their neural network.

xix. The origin of complex phyla not in sequence from simple to complex forms of vertebrates but all of a sudden during 20-million-year period of Cambrian Explosion totally disproves 'Darwin's Theory of Evolution and Natural Selection'.

\section{Scope for Future Research}

Of the four known forces of physics Electromagnetic force organized and prescribed life into existence. Electromagnetism does the programming and processing of the symphony of life, origin, evolution and reproduction. Sun light is an electromagnetic wave of light and particles.

Same planets occupy similar places in the zodiac of parents and children. Our genes ultimately come from an ancestral bacterium. This structure explained how cells copy their DNA. In other words, it revealed how parents 
make copies of their genes and pass them on to their children. Now, it requires to show which of such planets signify what identical bands in DNA Fingerprinting of parents and children pertaining to heredity, more particularly "Y" chromosome pertaining to patrilineal heredity.

The work of Jeffrey C. Hall, Michael Rosbash and Michael W. Young, the three 2017 Nobel Prize winners in Physiology or Medicine isolated a gene that controls the body's normal daily biological rhythm where Life on Earth is adapted to the rotation of our planet). In a similar way, Here also the question is whether Moon is responsible in triggering the hypothalamus.

The discoveries of Nobel Laureates Jeffrey C. Hall, Michael Rosbash and Michael W. Young explained the mechanism of working of our biological clock and its inner workings. This also explains how plants, animals and humans adapt their biological rhythm so that it is synchronized with the Earth's rotations. A synchronous relationship between the female human menstrual cycles and Lunar Rhythm exists. Since women adapt their biological rhythm of menstrual cycle once a month to the Moon's revolutions, the corresponding mechanism involved in the inner biological clock need to be discovered.

\section{Funding}

This research received no external funding.

\section{Conflicts of Interest}

The authors declare that they have no competing interests.

\section{Acknowledgements}

I profusely thank my son, Prof. Y. V. Ramana Rao, M. Tech., Ph. D, Department of Electronics and Communication Engineering, College of Engineering, Anna University, Chennai and my daughter Dr. Y. Rajalakshmi, M. Sc. (Mol. Bio.), Ph. D (Pathology) for their critical comments in improving this research paper.

\section{References}

[1] Harold FM (2005) Molecules into cells: specifying spatial architecture. Microbiol Mol Biol Rev 69: 544-564.

[2] McAdams HH, Shapiro L (2009) System-level design of bacterial cell cycle control. FEBS Lett 583: 3984-3991.

[3] Crick FHC, Orgel LE (1973) Directed panspermia. Icarus 19: 341-346.

[4] Hoyle F, Wickramasinghe NC (1980) Evolution from space. JM Dent, London.

[5] www.sciencealert.com/this-awesome-periodic-table.

[6] Professor Arjun Berera, news.com/astronomy/space-dust-biological. from the
University of Edinburgh's School of Physics and Astronomy, calculated how powerful flows of space dust could collide with particles in the atmospheric system of.

[7] climatica.org.uk/climate. Climate. Milankovitch-cycles: Introduction to Milankovitch cycles, and how they have controlled the Earth's climate over geological time.

[8] Nicola Scafetta (2014), The complex planetary synchronization structure of the Solar System, arXiv: 1405.0193v1 [physics. gen-ph] 16 Jan 2014.

[9] What Is it Called When All the Planets Are Lined Up in a sciencing.com/called-planets-lined-up-straight.

[10] Iyengar, R. N (2009) Ma'Sar, 9th century; Temple, 1998.

[11] Yarus M, Caporaso JG, Knight R. 2005, Origins of the genetic code: the escaped triplet theory. Annu Rev Biochem 74: 179198.

[12] Root-Bernstein RS. 1982b, On the origin of the genetic code. J Theor Biol 94: 895-905.

[13] Subba Rao, Y. V., Ramana Rao, Y. V. (2017) DNA Finger Printing and Horoscopy for Determining Paternity or Maternity of a Child, International Journal of Statistics and Actuarial Science. Vol. 1, No. 3, 2017, pp. 87-91).

[14] Gauquelin 91982), noted in The Cosmic Clocks, "if a child is born on a [magnetically] disturbed day, the number of heredity similarities is twice as high as on quiet days. This suggests that the Moon and planets do affect life, through the solar field," p. 127.

[15] Rao, Y. V. S, (Nov 2014), Natal Charts vis-a-vis DNA Entries Journal of Advances in Philosophy Online Journal -www.cirworld.com, Vol. No. 1, Page 14-19.

[16] Venkata Subba Rao Yeragudipati. Lunar Magnetism Orchestrates Menstrual Cycle in Symphony of Life. American Journal of Biomedical and Life Sciences. Vol. 7, No. 5, 2019, pp. 104-109. doi: 10.11648/j.ajbls.20190705.12.

[17] Callahan, (Aug 9, 2011), DNA Blocks can be made in Space NASA, www.defenceweb.co.za/joint/science-a-defense. Proceedings of the National Academy of Sciences of the United States of America.

[18] What is Darwin's Theory of Evolution? - Anatomical Origins www.anatomicalorigins.com.

[19] Evolutionnews.org/2018/03/answering-simplistic.

[20] Oparin, A. The Origin of Life, Foreign Languages Publishing House (1924), Dover publication (1938).

[21] F. A. Brown, Jr. et al., "Comparison of Fluctuations in Cosmic Radiation and Organismic Activity during 1954, 1955, and 1956," Amer. J. Physiolo., Vol. 195, 1958, pp. 237-243.

[22] H. J. Eysenck and Nias (1982), Astrology: Science or Superstition? 1st Edition, SBN-13: 978-0312058067, ISBN-10: 0312058063, St Martins Pr; 1st edition (August 1, 1982).

[23] Seymour P (1990), Astrology: The Evidence of Science. Revised edition, Arkana, London.

[24] Percy Seymour, Cosmic Magnetism, Bristol, U.K.: Adam Hilger, 1986. health.howstuffworks.com/.../births-full-moon.htm. 
[25] The Italian Journal, Minerva Ginecol., [March 1997; 49 (3): 91-94].

[26] The Scandinavian Journal, Acta Obstet Gynecol Scand, [1986; 65 (1): 45-48].

[27] Joshi R, Bharadwaj A, Gallousis S, Matthews R., Labor ward workload waxes and wanes with the lunar cycle, myth or reality? Primary Care Update Ob Gyns. 1998 Jul 1; 5 (4): 184. 6.

[28] Encyclopaedia Britannica (1994; Macropaedia article on Animal Behaviour, p. 761).

[29] Christopher Harwood Astrology - A Magnetic Attraction, first published by The Astrological Journal, 2009 / The Astrological Association of Great Britain / 01.02.2017.

\section{Biography}

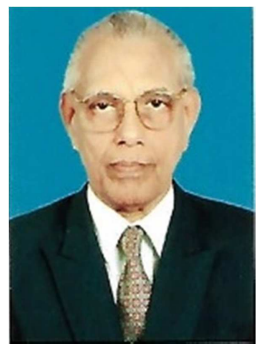

Venkata Subba Rao Yeragudipati, Ph.D., FIE., C. Eng. (I) was born in 1938. He is a Chartered Mining Engineer, Govt. of India Certified Mines Manager, Certified Mines Surveyor, Astronomer, Author and Astrologer. $\mathrm{He}$ was a faculty member of Mining Engineering Department of Osmania University, Hyderabad and later in Geology Department of S. V. University, Tirupati, India. After retirement, as Executive Engineer, he served as Visiting Professor of Jyotish (Astronomy), Rāshtriya Sanskrit Vidyāpeeth (University), Tirupati (2000-2013). He is the author of several books on Veda-Sciences and has several research papers to his credit. He is a Member of several professional bodies. 